\title{
BMJ Open Barriers and facilitators of diabetes management by continuous glucose monitoring systems among adults with type 2 diabetes: a protocol of qualitative systematic review
}

\author{
Mingyue Zheng (D) , ${ }^{1,2}$ Anamica Patel, ${ }^{3}$ Adeel Khoja, ${ }^{1}$ Yunting Luo, ${ }^{4,5}$ Wei Lin, ${ }^{6}$ \\ Qian $\mathrm{He},{ }^{7}$ Xuan Zhao, ${ }^{8}$ Juan Wang, ${ }^{9}$ Shenqiao $\mathrm{Yang}^{2}{ }^{2}$ Peng $\mathrm{Hu}^{2}$
}

To cite: Zheng M, Patel A, Khoja A, et al. Barriers and facilitators of diabetes management by continuous glucose monitoring systems among adults with type 2 diabetes: a protocol of qualitative systematic review. BMJ Open 2021;11:e046050. doi:10.1136/ bmjopen-2020-046050

- Prepublication history and additional supplemental material for this paper are available online. To view these files, please visit the journal online (http://dx.doi.org/10.1136/ bmjopen-2020-046050).

Received 19 0ctober 2020 Accepted 15 October 2021

Check for updates

(c) Author(s) (or their employer(s)) 2021. Re-use permitted under CC BY-NC. No commercial re-use. See rights and permissions. Published by BMJ.

For numbered affiliations see end of article.

Correspondence to

Mingyue Zheng;

mingyue.zheng@adelaide.edu. au and

Dr Peng $\mathrm{Hu}$;

hupeng@cdutcm.edu.cn

\section{ABSTRACT}

Introduction Studies suggest that continuous glucose monitors (CGMs) play an important role in the management of diabetes. Although general acceptance has been reported by patients with type 2 diabetes towards the use of CGMs, potential barriers exist like pain due to sensor insertion, accidental removal of the device or adhesive strip, impacts of daily activities, skin reactions to sensor adhesive, etc. This systematic review of qualitative studies aims to explore the perspectives, experiences and narratives of patients and caregivers about CGM use, and its barriers and facilitators.

Methods and analysis This review will include qualitative studies and cross-sectional and longitudinal cohort studies using open-ended questions, published in English by 30 0ctober 2021. The following electronic databases will be searched: Cochrane Library, PubMed, EMBASE, CINAHL, PsycINFO and Scopus. A search of grey literature will be conducted via an online search of Google Scholar, WorldCat, ClinicalTrials.gov and OpenGrey A combined search strategy using medical subject headings (MeSH), controlled vocabulary and 'free-text' terms will be appropriately revised to suit each database. Primary outcomes will include patient and caregiver perspectives on diabetes management regarding glucose control; living with CGM (quality of life, experience of wearing a CGM); psychological aspects (anxiety, depression, emotional burden); barriers (technical issues, financial issues) to use of CGM and thoughts (interpretation, understanding) on the CGM report. A qualitative meta-synthesis will be conducted employing a systematic literature search of existing literature, quality assessment using studyspecific tools and an aggregative thematic synthesis by a multidisciplinary team.

Ethics and dissemination Ethical approval is not required since this is a systematic review. The results will help improve clinical implementation of CGMs on part of both patients and caregivers.

PROSPERO registration number CRD42020152211.

\section{INTRODUCTION}

The prevalence of diabetes mellitus is predicted to increase to $10.9 \%$ (700 million)
Strengths and limitations of this study

- To the best of our knowledge, this is the first protocol of a systematic review conducted on qualitative research exploring the barriers and facilitators for diabetes management by CGMs amongst adults with type 2 diabetes.

- This study has the potential to contribute to the clinical uptake of CGMs by patients and their caregivers.

- The findings from this study will provide suggestions for patients and healthcare providers for better management of type 2 diabetes.

- A multidisciplinary review team will undertake independent and iterative thematic analysis of qualitative findings, interspersed with regular discussion and questioning of similar and contradictory findings with expertise in epidemiology, clinical nursing, biostatistics, dietetics and primary care prevention.

- As this is a secondary analysis, we will not have access to the full interview transcripts of the included studies; therefore, the analysis will be based on participant quotations selected by researchers to illustrate. As such, it may not entirely reflect the intended meaning or context.

by $2045 .^{1}$ The costs due to diabetes mellitus and its complications are also predicted to increase up to $\$ 2.1$ trillion by $2030 .^{2}$ Globally, around 1 in 11 adults have diabetes mellitus out of which $90 \%$ have type 2 diabetes mellitus (T2D). ${ }^{3}$ Therefore, it is essential to explore effective methods of diabetes management to alleviate the global health burden caused by T2D and its complications.

Continuous glucose monitor (CGM) is a device that can evaluate the blood glucose fluctuations in real time and can help develop personalised treatment plans to fully control short-term fluctuations in blood glucose levels. ${ }^{4}$ Many studies have shown its potential in diabetes management through 
identification of hyperglycaemia and hypoglycaemia, measuring glycaemic control and providing actionable information in the form of the CGM report to health carers and patients. ${ }^{5-7}$ Studies have also reported general acceptability by patients with T2D. ${ }^{8}$ It is estimated that real-time CGM can lead to higher quality-adjusted life years and reduced healthcare costs, with an expected incremental cost-effectiveness ratio of $€ 180553$ per quality-adjusted life year for patients with T2D. ${ }^{10}$ Therefore, CGM might be an effective method for T2D management. Even though the use of CGMs in adults with T2D is recommended by the endocrine society clinical practice guidelines, ${ }^{11}$ its uptake is less among people with T2D compared with people with type 1 diabetes (T1D).

Research amongst CGM using patients with T1D has identified several barriers to its use. A qualitative review exploring the impact and experience of using CGMs among people with T1D reported that CGM affects physical, emotional and relational aspects of life. It also found that clinicians can provide education and management to help reduce the barriers of CGM use. ${ }^{12}$ In 2018, a systematic appraisal of personal blogs analysing 39 blogs found that real-time CGM data sharing enhanced the feelings of safety among patients with T1D and their health carers. ${ }^{13}$ A recent review reported pain associated with insertion and wear of CGMs, body image issues, alarm fatigue, information overload, accuracy concerns, and clinical inertia to be barriers against CGM use among patients with T1D. ${ }^{14}$ CGMs have been widely used in the management of T1D, but their effectiveness has led to their use for management of T2D. Therefore, research needs to extend to exploration of experiences, barriers and facilitators to use of CGM among patients with T2D.

Effectiveness of CGM in T2D management has been reported through quantitative studies. ${ }^{510}$ However, only a few qualitative studies have explored its feasibility. ${ }^{15-17}$ In order to understand its wider acceptability, it is important to explore the narratives of people using CGM for management of T2D and their caregivers; and gain understanding of facilitators and barriers from the perspectives of both patients and caregivers. To improve its usage for clinical or family settings, CGM device functions are improved regarding user satisfaction and usability over time. ${ }^{12}$ It is suggested that use of CGM should consider the users' preferences and their assessment of acceptance. ${ }^{18}$ Therefore, both the experiences and thoughts of caregivers and users should be explored and generated among population with T2D.

Although general acceptance has been reported by patients with T2D towards the use of CGMs, potential barriers like pain due to sensor insertion, accidental removal of the device or the adhesive strip, impacts of sports and daily activities, skin reactions to sensor adhesive, lack of insurance coverage, etc exist. ${ }^{19}$ These barriers have been identified by qualitative studies, that also provide valuable perspectives for promoting the use of CGMs for clinical and self-management of diabetes. The studies also identified a gap in literature and a need for a meta-synthesis of qualitative studies on this topic. Therefore, this systematic review aims to explore the perspectives, lived experiences and narratives of patients and caregivers about their CGM use, and its barriers and facilitators.

\section{METHODS AND ANALYSIS Research questions}

This qualitative meta-synthesis aims to answer the following research questions:

1. How do people with T2D describe their experience (ie, glucose control, living with CGM, psychological aspects, understanding of CGM reports) of using CGM for managing their diabetes?

2. What are the views of people with T2D who use CGM for managing their diabetes about its effectiveness in maintaining glucose control?

3. What are the barriers and facilitators to CGM use reported by people with T2D and their caregivers; and what can the healthcare providers (ie, doctors, nurses, dietitians) do to address these barriers?

\section{Design and eligibility}

A qualitative meta-synthesis will be conducted for existing published and unpublished literature following a systematic literature search, quality assessment using studyspecific tools, aggregative thematic synthesis and the reporting of analytical themes to highlight the research questions in accordance with the guidelines of the Enhancing transparency in reporting the synthesis of qualitative research (ENTREQ). ${ }^{21}$ Preferred Reporting Items for Systematic Review and Meta-Analysis Protocols (PRISMA-P) (online supplemental file 1 ) $^{22}$ was used to prepare this protocol.

This appropriate structure of review is designed based on the Joanna Briggs Institute (JBI) template. It will include published and unpublished qualitative studies irrespective of the study design and cross-sectional and longitudinal cohort studies using open-ended questions that report perspectives and experiences of using CGM of patients with T2D and their caregivers. We will exclude studies of secondary designs (after checking the list of included studies for potential relevant primary studies), and studies only using quantitative methods. The inclusion of studies is primarily qualitative (ie, interviews, semistructured interviews, focus group discussions) studies and mixed-methods research (ie, questionnaires that are qualitative in nature with open-ended questions). The full list of inclusion and exclusion criteria has been presented in table 1.

\section{Information sources and search strategy}

A comprehensive PubMed search strategy was formulated after consultation with an experienced medical librarian (online supplemental file 2). Comprehensive search strategies for other databases will be developed using medical subject headings (MeSH), controlled vocabulary 


\begin{tabular}{|c|c|c|}
\hline Concepts & Inclusion & Exclusion \\
\hline Type of study & $\begin{array}{l}\text { Primary qualitative (ie, interviews, } \\
\text { semistructured interviews, focus group } \\
\text { discussion) studies } \\
\text { Mixed-methods research (ie, questionnaires of } \\
\text { qualitative nature with open-ended questions) }\end{array}$ & $\begin{array}{l}\text { Quantitative studies without qualitative component, } \\
\text { conference reports, commentaries, opinion pieces, } \\
\text { business reports, case reports, abstracts and systematic } \\
\text { reviews. Studies adopting mix-methods but only reporting } \\
\text { quantitative data }\end{array}$ \\
\hline Study population & $\begin{array}{l}\text { Age } \geq 18 \text { years; a confirmed diagnosis of T2D; } \\
\text { wearing any types of CGMs for at least } 3 \text { days }\end{array}$ & $\begin{array}{l}\text { Adolescents (under } 18 \text { years of age) and children; other } \\
\text { types of diabetes (ie, T1D, gestational diabetes mellitus) }\end{array}$ \\
\hline Language & English & Non-English \\
\hline CGM wearing & $\begin{array}{l}\text { Patients wearing any type of CGMs (ie, real- } \\
\text { time CGM, retrospective CGM, flash CGM) for } \\
\text { at least } 3 \text { days }\end{array}$ & Patients wearing CGMs for less than 3 days \\
\hline
\end{tabular}

*Inclusion will not be limited to designs such as phenomenological, grounded theory, ethnography, action and feminist research. CGM, continuous glucose monitor; T1D, type 1 diabetes; T2D, type 2 diabetes.

and 'free-text' words relevant to this review, and the logic grid will be developed with the research team (online supplemental file 2). Reference lists of relevant systematic reviews will be hand-searched to identify additional relevant studies or reviews. Topic experts in the field will also be contacted to identify any unpublished or ongoing work. Additional literature will be sought by checking the reference lists of journal and conferences articles, and checking cited articles. The research team will re-run the searches before the final systematic review will be published to ensure that they have not missed the latest studies.

The search strategy will aim to find both published and unpublished studies in English language published up to 30 October 2021 from the following databases: Cochrane Library, PubMed, EMBASE, CINAHL, PsycINFO and Scopus. To find unpublished grey literature (ie, theses and dissertations), Google Scholar, WorldCat, ClinicalTrials.gov and OpenGrey will be searched.

\section{Data management}

The reference management system EndNote V.X9 will be used to manage studies exported from all the sources. Two reviewers (MZ and AP) will independently and in duplicate screen the records for inclusion at the title/ abstract and full-text level screening via Covidence online system (https://www.covidence.org). Any disagreements at any screening level will be resolved through discussion. For any unresolved decisions, a third reviewer (AK) shall be consulted.

\section{Study selection and management}

The study selection will be done with Covidence, and includes title and abstract screening and full-text screening and extraction. Disagreements will be resolved by third and fourth reviewers (AK and YL) through discussion. A PRISMA flow diagram will be formed to record the details of the study selection process ${ }^{23}$ (online supplemental file 3 ).
Data on study characteristics will be extracted into an adopted JBI data extraction form by two reviewers (MZ and AP) and will include first author, year of publication, country, research questions, study population (age, ethnicity, gender, socioeconomic status), number of participants, study setting, data collection method, data analysis method, themes relevant to the objectives of this review and study limitations. Authors will be contacted via email to obtain relevant additional or missing information if required.

The analysis of included studies will follow the Standards for Reporting Qualitative Research: A Synthesis of Recommendations with 21 items. ${ }^{24}$ The thematic framework analysis approach will be adopted for data synthesis and analysis. ${ }^{25}$ The research team will follow the six stages of framework synthesis using NVivo (V.12.0.0 Plus): (a) procedure for analysis; (b) familiarisation with the interview; (c) coding; (d) developing a working analytical framework; (e) applying the analytical framework and (f) charting data into the framework matrix. Initial coding of a sample set (20\%) of papers will be conducted by two reviewers (MZ and $\mathrm{AP}$ ) independently and in consultation with a third reviewer (AK) to agree on the outline of the coding framework. Following this, coding of the remaining studies will be undertaken in duplicate with regular discussion to review and agree on additions and amendments to the framework.

The extracted data will be analysed line by line, and the identity of each first-order or second-order structure will be coded accordingly. The code will be inductively created by two independent reviewers for the response to uncovered findings, with a third reviewer's consultation. The two reviewers (MZ and AP) will then organise an expert meeting with team members to finalise the theme names and the structure/hierarchy of codes/ themes. The thematic analysis will be included but not limited to the following categories (online supplemental file 4), the final themes will be decided after the expert meeting based on the evidence available as it will follow 
an inductive analysis approach. The goal of this phase is to go beyond the initial findings to generate more understanding and information for future studies.

\section{Quality assessment}

Critical Appraisal Skills Programme (CASP) tool for mixed-methods studies (https://casp-uk.net/casp-toolschecklists/) will be used to critically appraise the quality of included studies using mixed-methods. This 10-item CASP tool is considered to be the most suitable tool to consider the quality parameters of qualitative work, and is a well-accepted and validated tool..$^{26} \mathrm{JBI}$ Critical Appraisal Checklist for Qualitative Research will be used to critically appraise the quality of included qualitative studies. ${ }^{27}$ Two independent reviewers ( $\mathrm{MZ}$ and $\mathrm{AP}$ ) will conduct the critical appraisal independently and in duplicate of all studies included for full text. If required, a third reviewer (AK) will identify the differences during risk of quality assessment. Studies will not be excluded based on their quality rating unless there are serious concerns (ie, very poor quality due to lack of transparency in reporting of methods or lack of reporting of participant quotations).

\section{Data synthesis and thematic analysis}

The strategy for the data synthesis of this review is based on the System for the Unified Management, Assessment and Review of Information (SUMARI) with the metaaggregation approach. ${ }^{28} \mathrm{~A}$ preliminary and comprehensive synthesis will be formed for the following aspects ${ }^{29}$ : textual descriptions of studies, groupings and clusters, tabulation, transforming data into a common rubric, vote counting as the descriptive tool, translating data for thematic and content analysis.

The narrative synthesis of the review, tables and narrative summaries of each included study will be illustrated by ATLAS.ti, data analysis software, following the Guidance of Narrative Synthesis in Systematic Reviews. ${ }^{29}$ This will involve a summary or synthesis of discoveries to concatenate the discoveries by combining the discoveries and categorising them based on similarities to generate a set of statements representing the set. These categories will then be subjected to a synthesis in order to produce a single comprehensive set of synthesised findings that can be used as a basis for evidence-based practice. Where textual pooling is not possible, the findings will be presented in narrative form.

Primary outcomes will include patient and caregiver perspectives on diabetes management regarding glucose control; living with CGM (quality of life, experience of wearing CGM devices); psychological aspect (anxiety, depression, emotional burden); barriers (technical issues, financial issues) of CGM use and thoughts (interpretation, understanding) on the CGM report. The outcomes will be modified depending on the evidence available. Secondary outcomes will include weight management, change in blood pressure and lipid profile.

To consider the impact on different systems, we will categorise different types of CGMs for additional analysis: (1) real-time CGM, (2) professional CGM and (3) flash/intermittently scanned CGM. In our report, we will tabulate summaries of the characteristics of the included studies. Important limitations and gaps within the evidence base will be presented and discussed.

\section{Patient and public involvement}

Patients and the general public will not be involved directly in the design and conduct of this review. However, the development of the review questions was informed by patient safety concerns and the experience of health professionals using eHealth applications in clinical practice. All data analysed during this study are included in the manuscript and additional files.

\section{Ethics and dissemination}

Ethical approval is not required as this systematic review will use secondary data. This systematic review protocol is registered in the International Prospective Register of Systematic Reviews (http://www.crd.york.ac.uk/PROSPERO). The results will help improve clinical implementation of CGMs for both patients and caregivers. This systematic review will be published in a peer-reviewed journal and presented in scientific conferences.

\section{DISCUSSION}

To the best of our knowledge, this is the first systematic review synthesising qualitative evidence exploring barriers and facilitators to CGM use among patients with T2D and their caregivers through exploration of their narratives of their perspectives and experiences. To ensure reliability, robustness and transparency of the predefined methods to answer the research questions of this review, we have referred to the SUMARI, JBI and ENTREQ guidelines. ${ }^{21} 2728$ Thematic analysis can identify prominent topics and process the literature on these topics in an organised and structured manner. It is flexible, allows reviewers considerable freedom, and is a method of integrating qualitative and quantitative evidence. ${ }^{30}$ Moreover, thematic analysis can be either theory driven (oriented to the appraisal of specific themes by interrogation of the study) or data driven (driven by the themes identified in the study itself) ${ }^{30}$ This review will adopt the data-driven thematic analysis to explore and analyse ${ }^{25}$ patients' and caregivers' experiences.

The results will be made available through publication in a peer-reviewed journal and through local and international presentations. This study will help identify barriers and facilitators to CGM use from the existing literature and provide scientific evidence for future studies regarding clinical use of CGMs for patients, caregivers and healthcare providers. This review will also provide future directions for observational and experimental studies regarding T2D management by avoiding issues resulting from the use of different types of CGMs. The findings of this qualitative evidence synthesis may help to explain why and how some interventions to improve CGM uptake 
are more effective than others. Knowledge of perceived facilitators and barriers to CGM use may generate insights about potential causal factors that affect impact. By identifying influential factors, it may also contribute to the development of more relevant, acceptable and effective interventions in the future. The findings can also be used to inform the design of future effectiveness reviews suggesting outcomes that are important to patients with T2D and their caregivers, as well as generating hypotheses that can be tested out, for example, in future subgroup analyses. In addition, the results from this synthesis may help improve our understanding of the reasons for acceptance of CGMs from the perspective of patients with T2D and their caregivers, contributing to the future development of more relevant, acceptable, and in turn, effective interventions to promote patient acceptance and uptake of CGMs.

\section{Author affiliations}

${ }^{1}$ Adelaide Medical School, The University of Adelaide Faculty of Health and Medical Sciences, Adelaide, South Australia, Australia

${ }^{2}$ School of Health and Rehabilitation, Chengdu University of Traditional Chinese Medicine, Chengdu, China

${ }^{3}$ Observatory Evidence Service, Public Health Wales, Cardiff, UK

${ }^{4}$ Center of Infectious Diseases, Sichuan University West China Hospital, Chengdu, Sichuan, China

${ }^{5}$ Institute for Disaster Management and Reconstruction, Sichuan University, Chengdu, Sichuan, China

${ }^{6}$ School of Management, Chengdu University of Traditional Chinese Medicine, Chengdu, Sichuan, China

${ }^{7}$ Department of Tendon Trauma, N0.1 Orthopedics Hospital of ChengDu QingYang District Medical Center, Chengdu, China

${ }^{8}$ School of Pharmacy, Chengdu University of Traditional Chinese Medicine, Chengdu, Sichuan, China

${ }^{9}$ School of Public Health, Chengdu University of Traditional Chinese Medicine, Chengdu, Sichuan, China

\section{Twitter Mingyue Zheng @Mingyue Zheng and Adeel Khoja @adeel.khoja}

Acknowledgements We delegate our thanks to Robert Franchini, the university librarian at the University of Adelaide for his assistance and guidance on developing search strategies.

Contributors MZ and PH conceived and designed the study. MZ drafted the manuscript and is the guarantor of the systematic review. AP developed the research questions and study design. MZ and $\mathrm{YL}$ designed the tables for included studies and will evaluate the quality of included studies in the systematic review. AP, MZ, YL, AK and XZ developed search strategy and will conduct data extraction and synthesis. QH contributed to the introduction part and additional files. WL, JW and XZ modified the methods and analysis part. PH, SY, AP and AK extensively revised the manuscript. All authors reviewed and approved the final manuscript as submitted and agreed to be responsible for all aspects of the work.

Funding This protocol was supported by Science and Technology Department of Sichuan Province (No. 2019132) and Chengdu University of Traditional Chinese Medicine (No. ZRQN201909) held by MZ.

Competing interests None declared.

Patient consent for publication Not required.

Provenance and peer review Not commissioned; externally peer reviewed.

Supplemental material This content has been supplied by the author(s). It has not been vetted by BMJ Publishing Group Limited (BMJ) and may not have been peer-reviewed. Any opinions or recommendations discussed are solely those of the author(s) and are not endorsed by BMJ. BMJ disclaims all liability and responsibility arising from any reliance placed on the content. Where the content includes any translated material, BMJ does not warrant the accuracy and reliability of the translations (including but not limited to local regulations, clinical guidelines, terminology, drug names and drug dosages), and is not responsible for any error and/or omissions arising from translation and adaptation or otherwise.

Open access This is an open access article distributed in accordance with the Creative Commons Attribution Non Commercial (CC BY-NC 4.0) license, which permits others to distribute, remix, adapt, build upon this work non-commercially, and license their derivative works on different terms, provided the original work is properly cited, appropriate credit is given, any changes made indicated, and the use is non-commercial. See: http://creativecommons.org/licenses/by-nc/4.0/.

ORCID iD

Mingyue Zheng http://orcid.org/0000-0002-7946-8911

\section{REFERENCES}

1 Saeedi P, Petersohn I, Salpea P, et al. Global and regional diabetes prevalence estimates for 2019 and projections for 2030 and 2045: Results from the International Diabetes Federation Diabetes Atlas, $9^{\text {th }}$ edition. Diabetes Res Clin Pract 2019;157:107843.

2 Bommer C, Sagalova V, Heesemann E, et al. Global economic burden of diabetes in adults: projections from 2015 to 2030. Diabetes Care 2018;41:963-70.

3 Zheng Y, Ley SH, Hu FB. Global aetiology and epidemiology of type 2 diabetes mellitus and its complications. Nat Rev Endocrinol 2018;14:88-98.

4 Dandona P. Minimizing glycemic fluctuations in patients with type 2 diabetes: approaches and importance. Diabetes Technol Ther 2017;19:498-506.

5 Vigersky R, Shrivastav M. Role of continuous glucose monitoring for type 2 in diabetes management and research. J Diabetes Complications 2017;31:280-7.

6 Rodbard D. Continuous glucose monitoring: a review of recent studies demonstrating improved glycemic outcomes. Diabetes Technol Ther 2017;19:S-25-S-37.

7 Dungan K, Verma N. Monitoring technologies-continuous glucose monitoring, mobile technology, biomarkers of glycemic control. In: Endotext [Internet]. MDText. com, Inc, 2018.

8 Kompala T, Neinstein A. A new era: increasing continuous glucose monitoring use in type 2 diabetes. Am J Manag Care 2019;25:123-6.

9 Carlson AL, Mullen DM, Bergenstal RM. Clinical use of continuous glucose monitoring in adults with type 2 diabetes. Diabetes Technol Ther 2017;19:S-4-S-11.

10 García-Lorenzo B, Rivero-Santana A, Vallejo-Torres L, et al. Costeffectiveness analysis of real-time continuous monitoring glucose compared to self-monitoring of blood glucose for diabetes mellitus in Spain. J Eval Clin Pract 2018;24:772-81.

11 Peters AL, Ahmann AJ, Battelino T, et al. Diabetes technologycontinuous subcutaneous insulin infusion therapy and continuous glucose monitoring in adults: an endocrine society clinical practice guideline. J Clin Endocrinol Metab 2016;101:3922-37.

12 Messer LH, Johnson R, Driscoll KA, et al. Best friend or spy: a qualitative meta-synthesis on the impact of continuous glucose monitoring on life with type 1 diabetes. Diabet Med 2018;35:409-18.

13 Litchman ML, Allen NA, Colicchio VD, et al. A qualitative analysis of real-time continuous glucose monitoring data sharing with care partners: to share or not to share? Diabetes Technol Ther 2018;20:25-31.

14 Stone JY, Bailey TS. Benefits and limitations of continuous glucose monitoring in type 1 diabetes. Expert Rev Endocrinol Metab 2020;15:41-9.

15 Allen N, Whittemore R, Melkus G. A continuous glucose monitoring and problem-solving intervention to change physical activity behavior in women with type 2 diabetes: a pilot study. Diabetes Technol Ther 2011;13:1091-9.

16 Mattishent K, Lane K, Salter C, et al. Continuous glucose monitoring in older people with diabetes and memory problems: a mixedmethods feasibility study in the UK. BMJ Open 2019;9:e032037.

17 Allen NA, Jacelon CS, Chipkin SR. Feasibility and acceptability of continuous glucose monitoring and accelerometer technology in exercising individuals with type 2 diabetes. J Clin Nurs 2009;18:373-83.

18 Steed L, Cooke D, Hurel SJ, et al. Development and piloting of an acceptability questionnaire for continuous glucose monitoring devices. Diabetes Technol Ther 2008;10:95-101.

19 Hirsch IB. Professional flash continuous glucose monitoring as a supplement to A1C in primary care. Postgrad Med 2017;129:781-90.

20 Patton SR. Adherence to glycemic monitoring in diabetes. J Diabetes Sci Technol 2015;9:668-75. 
21 Tong A, Flemming K, Mclnnes E, et al. Enhancing transparency in reporting the synthesis of qualitative research: ENTREQ. BMC Med Res Methodol 2012;12:181.

22 Moher D, Shamseer L, Clarke M, et al. Preferred reporting items for systematic review and meta-analysis protocols (PRISMA-P) 2015 statement. Syst Rev 2015;4:1.

23 Moher D, Liberati A, Tetzlaff J, et al. Preferred reporting items for systematic reviews and meta-analyses: the PRISMA statement. PLoS Med 2009;6:e1000097.

24 O'Brien BC, Harris IB, Beckman TJ, et al. Standards for reporting qualitative research: a synthesis of recommendations. Acad Med 2014;89:1245-51.

25 Thomas J, Harden A. Methods for the thematic synthesis of qualitative research in systematic reviews. BMC Med Res Methodol 2008;8:1.

26 Long HA, French DP, Brooks JM. Optimising the value of the critical appraisal skills programme (CASP) tool for quality appraisal in qualitative evidence synthesis. Research Methods in Medicine \& Health Sciences 2020;1:31-42. 23.

27 Joanna Briggs Institute,. JBI critical appraisal checklist for qualitative research, 2017. Available: http://joannabriggs.org/assets/docs/ criticalappraisal-tools/JBI_Critical_Appraisal-Checklist_for_ Qualitative_Research2017. pdf

28 Piper C. System for the unified management, assessment, and review of information (SUMARI). Journal of the Medical Library Association 2019;107:634.

29 Popay J, Roberts H, Sowden A. Guidance on the conduct of narrative synthesis in systematic reviews. A Product from the ESRC Methods Programme Version 2006;1:b92.

30 Dixon-Woods M, Agarwal S, Jones D, et al. Synthesising qualitative and quantitative evidence: a review of possible methods. $J$ Health Serv Res Policy 2005;10:45-53. 